\title{
ALCUNE RIFLESSIONI SULLA BIBLIOGRAFIA GEOGRAFICA DELLA REGIONE ITALIANA (ANNI 1991-1992)
}

NICOLETTA VARANI ${ }^{12}$

ANTONELLA PRIMI ${ }^{2}$

\section{INTRODUZIONE}

Il contenuto dell'ultimo volume della Bibliografia Geografica della Regione Italiana induce a compiere riflessioni sulle recenti tendenze emerse nella geografia italiana.

Da sempre i geografi hanno manifestato interesse per l'organizzazione del territorio mettendo a punto analisi ambientali sempre più articolate e approntando le metodologie. La ricerca geografica in questi ultimi decenni si è andata integrando con quella di altre scienze, fisiche e umane, interessate allo studio del territorio. Durante questo percorso la geografia si è inoltrata verso una diversificazione di interessi travalicando, a volte, i propri confini e addentrandosi nei campi di altre discipline, con il rischio di mettere in crisi la propria unità scientifica.

La produzione geografica dei primi anni '90 fa ritenere che questa fase sia stata superata e che la geografia abbia riscoperto una propria originalità di produzione e conoscenza e abbia ricercato unità mettendo a punto metodologie comuni a tutti gli indirizzi, fisici e umani.

La produzione geografica a cui si fa riferimento è stata circoscritta allo spazio nazionale, in linea con la tradizione delle bibliografie della Società Geografica Italiana. In tal modo, dallo spoglio dei contributi vagliati, è emerso che, accanto ai classici temi della geografia storica, fisica, economica, urbana e politica, sono stati sviluppati studi sempre più mirati nel campo della geografia del turismo e della geografia medica e si è manifestata una maggiore sensibilità per i temi della qualità della vita e della tutela dell'ambiente.

Il panorama della produzione consente di ripartire la bibliografia in 18 settori, a loro volta suddivisi in 61 sezioni più specifiche e particolareggiate (fig. 1).

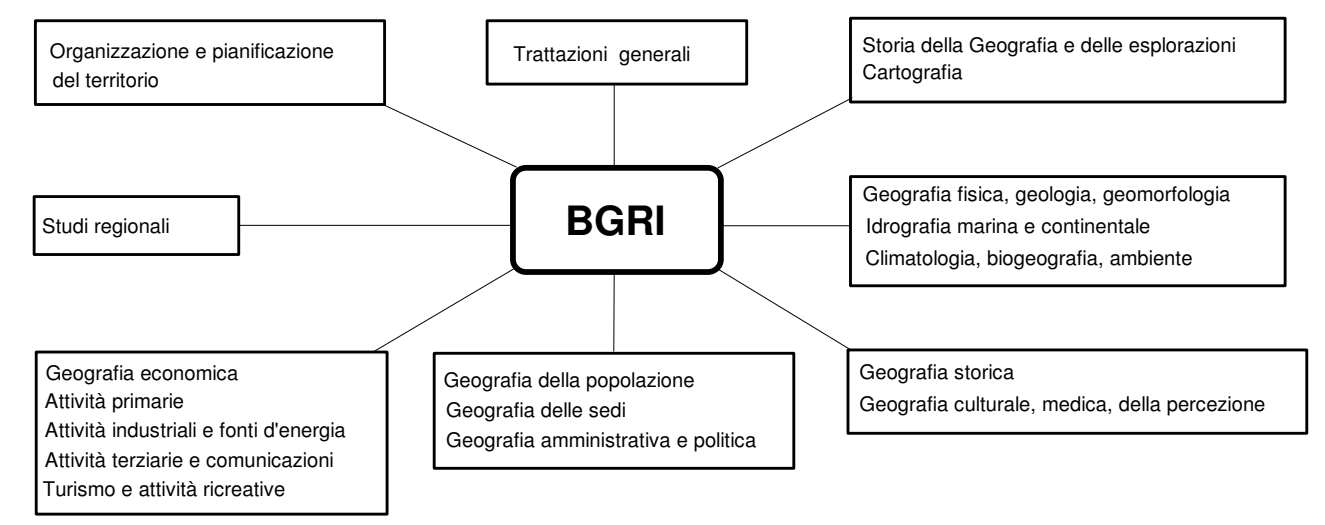

Fig. 1 - I settori della Bibliografia Geografica della Regione Italiana

Figura 1 - Sectores da Bibliografia Geográfica

\footnotetext{
${ }^{1}$ Sebbene il contributo sia stato preparato come una singola entità i paragrafi $1,3,5$, sono stati redatti da Nicoletta Varani e i paragrafi 2 e 4 da Antonella Primi.

2 Docentes da Universidade de Génova. Instituto di Scienze Geografiche. Fax: (39 - 10) 2095347.
} 


\section{1- LA PRODUZIONE GEOGRAFICA DEI PRIMI ANNI '90}

La produzione geografica del primo biennio degli anni '90, esaminata nella bibliografia, è costituita da 980 contributi tra volumi ed articoli reperiti su segnalazione degli stessi autori e attraverso la consultazione delle principali riviste, dei periodici e dei cataloghi delle case editrici.

Tra i settori più ricchi di contributi ve ne sono alcuni tradizionali, quali le trattazioni generali, la storia della geografia e delle esplorazioni, la cartografia, la geografia fisica, la geografia delle sedi, gli studi regionali e l'organizzazione del territorio, ed alcuni caratterizzati da temi considerati "emergenti", quali le problematiche ambientali, la geografia del turismo e la geografia medica (fig. 2).

Le trattazioni generali comprendono studi di epistemologia che presentano una riflessione sul metodo, le caratteristiche e le finalità dell'analisi geografica. I temi maggiormente approfonditi riguardano l'evoluzione della geografia e pongono l'accento sulla "varietà delle geografie" (CORNA PELLEGRINI e BIANCHI, 1992), sulla "diversità della geografia" (COPPOLA, 1992) e sulle principali categorie concettuali, ad esempio il rapporto spazio-tempo, la regione e il paesaggio (BUZZETTI, 1992). Il quadro è completato dagli studi sull'epistemologia esterna, sul rapporto tra geografia e altre discipline, tra cui l'ecologia, la bioetica (DA POZZO, 1992) e la storia e, infine, da studi specificatamente metodologici. Accanto ad essi, vanno ricordati i manuali, spesso frutto della collaborazione di più autori, che affrontano in modo sistematico $\mathrm{i}$ grandi temi della geografia umana, regionale e urbana; anche le raccolte di scritti e le ricerche relative all'Italia nel suo complesso rispecchiano le problematiche epistemologiche, metodologiche e i principali filoni di ricerca.

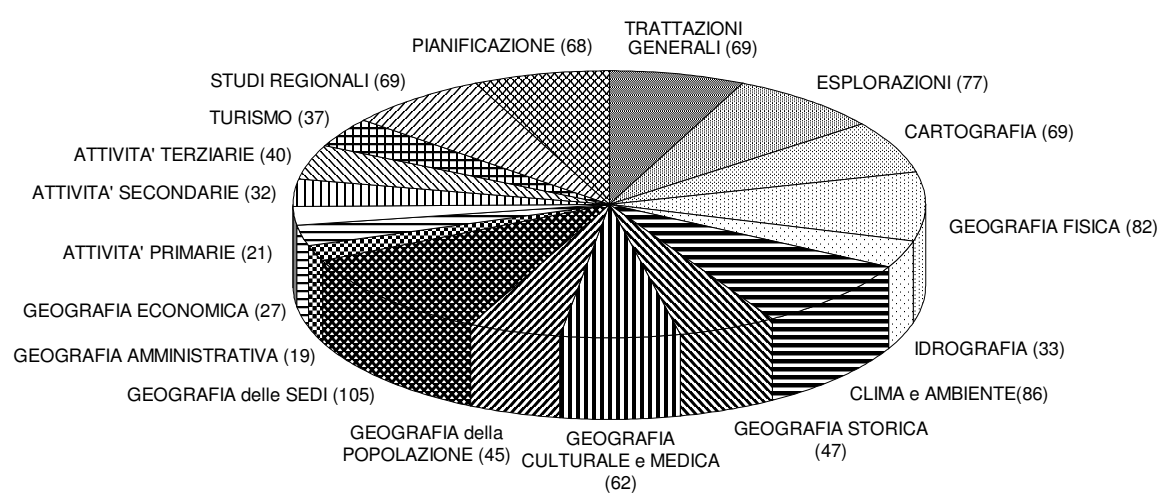

Fig. 2 - La produzione geografica dei primi anni '90

Figura 2 - A produção geográfica do início dos anos 90

Nel settore dedicato alla storia della geografia e delle esplorazioni geografiche risulta molto ricca la sezione riservata agli studi colombiani elaborati in occasione delle celebrazioni per il quinto centenario della scoperta dell'America. L'argomento viene affrontato da molteplici punti di vista: studi di carattere generale sulla figura di Colombo e i suoi viaggi, contributi che ripercorrono la formazione culturale del navigatore e la cultura dell'epoca, ed analisi delle conoscenze geografiche e naturalistiche conseguenti alla scoperta. L'impresa colombiana viene esaminata sia prendendo in considerazione le motivazioni, la genesi e l'evoluzione del progetto di Colombo sia ricostruendo le vicende dei suoi quattro viaggi. Il quadro è completato da contributi dedicati ai paesi in cui il navigatore ha vissuto per alcuni periodi della sua 
vita, ad esempio la Liguria e il Portogallo, e da lavori di carattere critico-interpretativo sulla cartografia e la documentazione colombiana.

La cartografia è stata approfondita sia attraverso la prospettiva storica sia mediante studi di cartografia moderna, tra i quali spiccano opere sulla costruzione e l'interpretazione delle carte, sul valore della cartografia e i suoi rapporti con la geografia. Particolare interesse hanno suscitato anche la cartografia tematica e i più recenti ausili informatici per la cartografia e la geografia quali il telerilevamento e i GIS (Geographical Information Systems).

La geografia fisica, la geologia e la geomorfologia sono state accorpate nel medesimo capitolo della bibliografia poiché, a seguito della grande specializzazione raggiunta da queste discipline, l'interesse per tali studi da parte dei geografi umani è diminuito nel corso degli anni. La progressiva specializzazione della geografia fisica ha favorito la costituzione di un vero e proprio gruppo di ricerca e la nascita (1978) della rivista "Geografia Fisica e Dinamica Quaternaria" su cui vengono pubblicati i principali studi del settore. I contributi raccolti, prevalentemente a carattere descrittivo ed applicativo, affrontano una grande varietà di temi che delineano un quadro preciso degli attuali indirizzi della ricerca.

In questo ambito rientrano pure gli studi di idrografia marina e continentale tra cui emergono le indagini sulla gestione e la conservazione delle risorse idriche, importanti fattori nella distribuzione degli insediamenti e delle attività umane.

La climatologia, in quanto studio dei vari tipi di clima e delle regioni climatiche e delle influenze che i climi esercitano sull'ambiente e sulle forme biotiche, è stata inserita nel settore in cui compaiono anche la biogeografia e le problematiche ecologiche ed ambientali.

Alle finalità e alle metodologie d'indagine della geografia storica, ormai consolidate da una lunga tradizione di ricerca, negli anni considerati non vengono dedicate opere sistematiche mentre la prospettiva storica si esprime soprattutto nello studio delle fonti, dell'evoluzione del popolamento, degli insediamenti e dell'organizzazione territoriale.

I contributi di geografia culturale si dedicano all'esame dei rapporti tra cultura e territorio, soprattutto a quello delle influenze culturali in campo ambientale. Ai contributi specifici sulla cultura urbana si affiancano quelli condotti a scala regionale sul valore culturale degli spazi agricoli e sui beni culturali. Numerosi sono gli studi di geografia medica, che comprende contributi sia di impostazione puramente teorica ed epistemologica sia analisi dedicate a specifici ambienti o patologie. Per quanto concerne le minoranze etnico-linguistiche alcuni studi riguardano problematiche di comunità italiane all'estero, gruppi etnici minoritari nel Mezzogiorno o l'integrazione di vari gruppi etnici nelle regioni italiane. Anche se in Italia non è ancora pienamente legittimata, un cenno va fatto alla geografia del genere che comincia a suscitare interesse tra i geografi umani. Alla geografia della percezione appartengono alcuni contributi che affrontano l'argomento con riferimento alla geografia della popolazione, alla percezione spaziale della cultura e degli edifici religiosi, nella prospettiva di eventuali strategie di intervento e in chiave storica attraverso un esame di fonti letterarie e cartografiche.

Nell'ambito della geografia della popolazione appaiono ampie e dettagliate opere sistematiche sulle ricerche di demografia, sui rapporti tra gruppi umani e territorio e sull'evoluzione demografica delle città italiane. Altri contributi affrontano specifiche tematiche come l'esplosione demografica del XX secolo, le dinamiche demografiche e i movimenti pendolari. 
La geografia delle sedi è il settore più ricco di contributi (105). Infatti nel primo biennio degli anni '90 la letteratura si è arricchita di numerosi studi che hanno approfondito le tipologie, l'organizzazione e l'evoluzione degli insediamenti urbani. Il fenomeno urbano (DEMATTEIS, 1992) viene analizzato tenendo conto degli aspetti specifici della situazione italiana. Ampi studi sono dedicati ai sistemi urbani (Bertuglia e LA Bella, 1991) attraverso un esame della loro delimitazione, delle reti e trame urbane e delle connessioni con l'innovazione territoriale. L'urbanizzazione viene esaminata da studi che evidenziano le diverse dinamiche che la caratterizzano. In particolare ampie e articolate ricerche sono dedicate al decentramento urbano e al fenomeno della controurbanizzazione analizzata dal punto di vista teorico e nel contesto di importanti aree urbane. Non mancano contributi che hanno preso in considerazione le dinamiche delle aree periferiche, periurbane e dello spazio rururbano, soprattutto attraverso l'esame degli spostamenti residenziali come pure studi sulle aree di attrazione e le agglomerazioni urbane. I contributi dedicati alle aree metropolitane esaminano, da un lato, i principi e le metodologie utilizzate per la loro delimitazione e dall'altro lato le funzioni delle aree metropolitane italiane. Nel quadro degli studi di geografia delle sedi rientrano le monografie di città, che analizzano la struttura e lo sviluppo urbano, cui si aggiungono analisi più ampie sulle caratteristiche e sulle dinamiche insediative del fenomeno urbano in ambito provinciale e regionale con speciale considerazione per il Mezzogiorno. Molti contributi sono riservati all'analisi degli insediamenti storici e rurali e altri ancora sono dedicati alle ville e alle residenze di campagna, in particolare dell'Italia centro-meridionale.

Per quanto concerne la geografia amministrativa e politica va sottolineato che $\mathrm{i}$ contributi di questo settore non sono numerosi ma testimoniano che, seppure modesto, esiste sempre un certo interesse per studi relativi a problemi di geografia amministrativa e a temi di geopolitica. Sono da segnalare, come aggiornamenti, studi sull'istituzione delle più recenti province italiane e sulla delimitazione comunale di grandi città e sulle modifiche dei confini, nonché studi di geografia elettorale.

La rassegna degli studi di geografia economica apparentemente può sembrare limitata. In realtà essi compaiono in molti dei settori della bibliografia che includono l'analisi delle tendenze evolutive di carattere economico e sociale. Una parte delle problematiche della geografia economica è riferita ai consueti settori delle attività primarie, delle attività industriali e fonti di energia e delle attività terziarie incluse le comunicazioni. In questo contesto il turismo e le attività ricreative risultano tra $\mathrm{i}$ temi definiti come emergenti.

Numerosi sono i temi della ricerca geografica, che trovano un riscontro anche dal punto di vista applicativo nell'organizzazione $e$ pianificazione territoriale. In particolare si rileva un crescente interesse per le analisi sull'impatto ambientale e per le problematiche scaturite dalla politica ambientale. In questo ambito emerge l'attenzione per lo studio degli aspetti geografici della gestione e della tutela dell'area costiera. L'apporto della geografia segue vari percorsi di indagine: da un contesto generale come quello della politica ambientale a quelli più tecnici della Valutazione di Impatto Ambientale (V.I.A.) e dell'istituzione delle aree protette. Premesso che non sono stati esaminati lavori di pianificazione prodotti da enti locali, diversificati risultano i contributi geografici nell'ambito regionale, i temi riguardano: la pianificazione agraria e i riordini fondiari, la gestione delle aree boschive, la politica urbana, la programmazione industriale e più in generale aspetti della pianificazione regionale e provinciale. 


\section{2- RECENTI TENDENZE DELLA RICERCA GEOGRAFICA}

Dai contributi esaminati è emerso con chiarezza un rilevante interesse da parte dei geografi per temi che, pur nella loro diversità, rispecchiano una nuova sensibilità nei confronti del binomio società-ecosistema. L'analisi dell'ambiente, la geografia del turismo e la geografia medica mettono in rilievo l'esigenza di individuare un metodo geografico per studiare il territorio e le sue continue trasformazioni.

Nei tardi anni '80 il quadro di riferimento del geografo è mutato: la dimensione ambientale, che il funzionalismo non aveva preso in considerazione, è stata introdotta nella ricerca, soprattutto in quella regionale.

All'inizio degli anni '90 numerosi contributi hanno prodotto una letteratura geografica sia considerando l'aspetto ecologico vero e proprio, sia attraverso la descrizione in termini di ecosistemi per giungere alla più recente produzione in termini di sviluppo sostenibile. Infatti la politica dello sviluppo sostenibile, venuta alla ribalta nel panorama mondiale con la Conferenza di Rio de Janeiro (1992), ha offerto ai geografi alcuni nuovi percorsi di ricerca. In quest'ottica i campi di analisi diventano molteplici e coinvolgono il geografo in nuove ricerche sui valori e sulle funzioni del territorio. Ad esempio il tema della Valutazione di Impatto Ambientale (V.I.A.) è divenuto di primaria importanza nell'ambito degli interventi per la salvaguardia ambientale e viene affrontato da un punto di vista descrittivo e critico mediante considerazioni sulla validità delle procedure di questa recente metodologia. Nell'ambito della geografia del mare a cui è stata dedicata un'intera sezione del XXVI Congresso Geografico Italiano (Genova, 4-9 maggio 1992), grande interesse hanno suscitato temi quali l'interazione tra comportamento umano e mare finalizzata allo studio degli usi e alla definizione di modelli di gestione (VALLEGA, 1992). Oggetto di analisi sono: le pressioni antropiche urbane, industriali e turistiche sulle fasce costiere (MINCA, 1991; ZUNICA, 1992), nonché la valutazione d'impatto ambientale, le risorse biologiche del mare, la salvaguardia dell'ambiente marino (SoDANO, 1992). Il grande interesse suscitato nell'ambito dell'istituzione e della tutela delle aree protette si riflette nei contributi che prendono in considerazione sia aspetti giuridici e gestionali a livello nazionale, sia quelli più specifici di alcuni casi di studio (SPOTO e FrANZOSINI, 1991; VARANI, 1991). Molti contributi dell'analisi geografica in questo settore hanno avuto come oggetto di studio aree marine e costiere di altri paesi del Mediterraneo per cui non rientrano nei contributi esaminati nel redigere la Bibliografia Geografica della Regione Italiana ma è doveroso sottolinearne l'importanza.

L'evoluzione della ricerca geografica fa sì che l'ambiente si proponga come un importante oggetto di studio che va percepito nella sua complessità, posto in relazione con la pressione esercitata dalla comunità umana e di cui il geografo non fornisce una descrizione, ma una conoscenza prodotta attraverso rappresentazioni.

Una considerazione a parte va fatta per tutti quei contributi di geografi che rientrano nella guida del TCI (1991) e forniscono una scheda contenente notizie sul paesaggio naturale e l'ambiente dei più interessanti itinerari, turistici e non, del territorio italiano.

Il geografo è approdato tardi allo studio del turismo e del tempo libero poiché per lungo tempo li ha considerati settori trascurabili e nelle monografie regionali li ha spesso confinati nel capitolo dedicato all'industria. I motivi di tale ritardo sono vari: la recente diffusione del turismo a lungo raggio e di quello di prossimità come fenomeno di massa; l'esiguità e l'attendibilità dei dati statistici e la maggiore difficoltà d'approccio nell'analisi delle attività ricreative rispetto a quelle economiche.

Inoltre, va sottolineato che prima della seconda guerra mondiale il turismo e il tempo libero hanno interessato solo ristrette fasce sociali. Dopo il conflitto la situazione è mutata: turismo e fenomeno della seconda casa hanno interessato in tutti i 
paesi industrializzati masse sempre più consistenti e il turismo ha cominciato ad essere sempre più legato alla congiuntura economica nazionale. Infatti, la forte crescita delle attività turistiche ha imposto l'analisi di una delocalizzazione e di una rilocalizzazione, nonché delle modalità di integrazione dei fenomeni turistici in una struttura regionale, con essi venuta spesso in conflitto.

Nei contributi esaminati emerge che le ricerche del settore vengono inserite nel quadro più ampio della geografia regionale e in settori più specifici come la geografia dei trasporti. Lo studio dei caratteri generali dell'attività turistica si basa prevalentemente sui temi culturali, sociali, economici e infine sulla ricerca di nuovi spunti di riflessione operativa nonché sulle dimensioni e gli aspetti qualitativi dell'offerta. Più specificatamente viene analizzato un particolare tipo di turismo venuto alla ribalta alla fine degli anni '80, l'agriturismo, cui sono dedicati vari lavori e un sostanzioso volume (BELLENCIN MENEGHEL, 1991), che mette bene in evidenza le caratteristiche e l'evoluzione di questo nuovo modo di fare turismo nelle varie realtà regionali, considerandone anche gli aspetti giuridici.

In conclusione emerge che seppure la geografia del turismo non verrà istituzionalizzata come una branca disciplinare autonoma avrà un ruolo sempre più importante nello studio delle tipologie e delle interdipendenze del turismo con i fattori economici, ambientali, culturali, sociali e politici.

Per anni la geografia medica non ha avuto in Italia molti cultori poiché è stata ritenuta una branca troppo specialistica. Infatti, il geografo per divenire geografo medico deve acquisire un complesso di informazioni di carattere medico, che gli consentano soprattutto di comprendere il linguaggio con il quale comunicare in modo interdisciplinare.

Agli inizi degli anni '80 grazie al primo Seminario Internazionale di Geografia Medica tenutosi a Roma con il patrocinio dell'UGI a cui ne ha fatto seguito un secondo nel 1985 a Cassino si è cominciato a dare più spazio a questa specializzazione della geografia. Nei primi anni '90 i geografi hanno prodotto numerosi studi di geografia medica; la parte più consistente dei contributi rientra negli atti del IV Seminario Internazionale di Geografia Medica. Si tratta di un'ampia pubblicazione che ha affrontato numerose problematiche relative alla salute, esaminandole in diversi contesti ambientali, ad esempio negli ecosistemi naturali, negli ecosistemi umanizzati e negli ecosistemi "artificiali". L'analisi è focalizzata sempre sul binomio uomo-ambiente. In realtà, la forma di equilibrio tra uomo e ambiente che viene identificata con la salute deve essere alla base di qualsiasi programma di intervento dell'uomo sul territorio. Anche gli studi sulla qualità della vita analizzata in rapporto all'ambiente e alla salute, o alle aree urbane e al traffico rivestono sempre maggior importanza nell'ambito della geografia. Inoltre, i geografi medici si occupano anche dello studio delle strutture sanitarie e non sono solo interessati alla distribuzione spaziale delle malattie, ma anche ai processi e ai percorsi attraverso i quali le malattie si trasmettono e si diffondono. La geografia medica deve essere considerata una disciplina geografica poiché adotta pienamente il metodo geografico che consiste nello studio dei fenomeni che nascono dal rapporto uomo-ambiente: solo che il primo termine del rapporto, l'uomo, è visto non solo come agente trasformatore ma anche come soggetto di malattia (PALAGIANO, 1987). 


\section{3- LA CONSISTENZA DELLA PRODUZIONE GEOGRAFICA}

I volumi pubblicati nel primo biennio degli anni '90 risultano essere circa un 1/4 rispetto alla totalità dei contributi comparsi su riviste, atti di convegni e opere miscellanee (fig. 3).

Dei 164 volumi, molto spesso opere di più autori o coordinate da un curatore, poco più di $1 / 3$ sono rappresentati da opere sistematiche. Sono state considerate tali tutte quelle opere che hanno fornito una classificazione organica e completa di una determinata tematica concernente i vari settori della geografia.

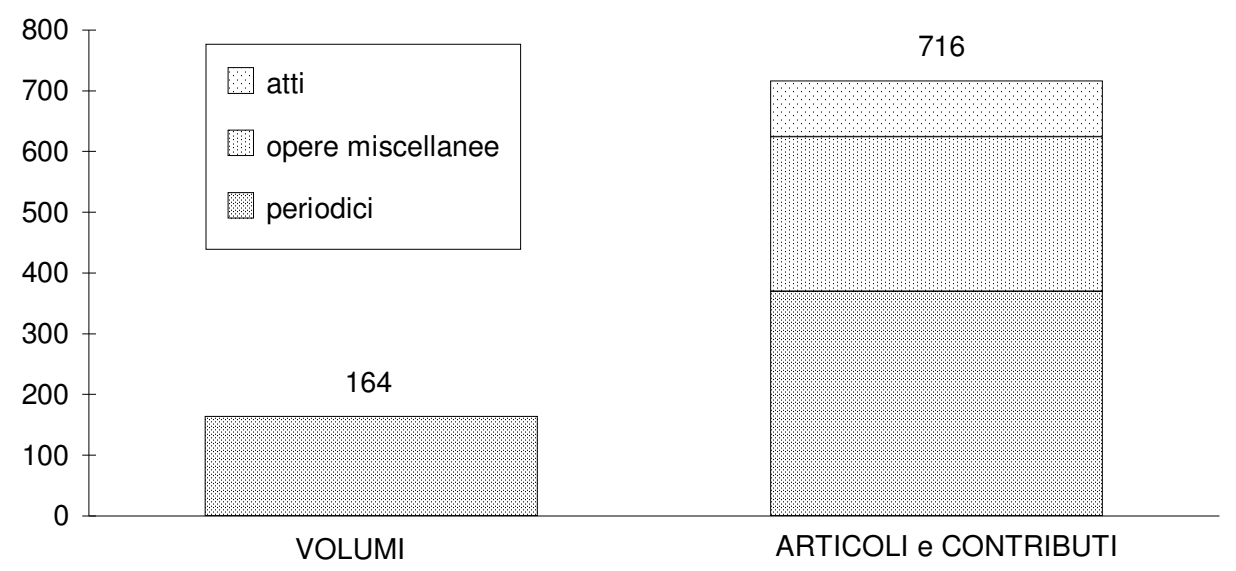

Fig. 3 - Rapporto fra volumi ed articoli nella produzione geografica degli anni 1991-1992

Figura 3 - Relação entre livros e artigos na produção geográfica dos anos 1991-1992

Nel capitolo dedicato alle trattazioni generali compare il maggior numero di opere sistematiche proprio perché vi rientrano veri e propri manuali e volumi metodologici e di epistemologia. Altre opere sistematiche si riscontrano in quei settori definiti "emergenti", come la geografia medica e in quelli più corposi come la geografia delle sedi e l'organizzazione e pianificazione del territorio.

Per quanto concerne i 716 articoli inseriti nella bibliografia, la parte più consistente (370) è stata pubblicata su periodici. Risultano nettamente prevalenti gli studi apparsi su riviste geografiche e settoriali, per lo più a carattere nazionale, ma talvolta a diffusione locale; mentre alcuni articoli (44) sono ospitati anche su riviste straniere. Accanto ad essi vanno ricordati i lavori (88) appartenenti alle pubblicazioni periodiche degli Istituti e dei Dipartimenti di geografia e a volumi speciali quali le Memorie della Società Geografica Italiana e le Memorie della Società Geologica Italiana.

Altri articoli (92) sono inclusi negli atti dei congressi; i contributi più numerosi si riferiscono al convegno sulla "cartografia geografica nel progresso delle conoscenze sull'Oriente" (BENCARDINO, 1991), alla conferenza internazionale sui "cambiamenti ambientali nelle aree carsiche" (SAURO e BONDESAN, 1991), al seminario internazionale sulla "geografia medica e gli ecosistemi" (PALAGIANO, DE SANTIS e SCIFONI, 1991), al convegno sulla "popolazione delle città italiane" (DISTASO, 1992), al congresso internazionale sulla "emigrazione e presenza italiana in Argentina" (CITAREllA, 1992) e agli "atti preparatori del XXVI Congresso Geografico Italiano" (A.Ge.I., 1992). Infine, va precisato che gli atti del convegno dedicato a "risorse idriche e organizzazione del territorio" sono stati pubblicati nel $4^{\circ}$ fascicolo (1992) della Rivista Geografica Italiana.

I restanti articoli (254) sono inseriti in volumi di più autori e in opere miscellanee, tra cui si ricordano gli studi in onore di Osvaldo BALDACCI (PALAGIANO e 
PARATORE, 1991), che riuniscono molti contributi che spaziano su di un ampio orizzonte geografico, gli studi in memoria di Giorgio VALUSSI (ORIOLES, 1992) e la ristampa di alcuni scritti di Bruno NICE (1992).

Gli studi regionali, importante settore della ricerca geografica, da sempre contribuiscono a delineare un quadro puntuale della realtà italiana. A livello regionale e locale (fig. 4) appaiono preponderanti i lavori relativi alla Toscana, tra i quali spiccano quelli inerenti la geografia fisica e la geomorfologia, le risorse idriche, il clima e l'ambiente naturale, la geografia storica, i paesaggi collinari e costieri e l'analisi dei vari aspetti economico-territoriali. Alla Liguria sono riferite sia opere che raccolgono contributi di più autori sia lavori di minore ampiezza a carattere prevalentemente fisico e storico nonché gli atti preparatori del XXVI Congresso Geografico Italiano ospitato nel 1992 a Genova. Molti aspetti del Veneto sono analizzati in studi per la maggior parte raccolti in un volume monografico che esamina attraverso varie prospettive molteplici aspetti di questa regione; nel complesso risultano preponderanti i lavori appartenenti alla geografia delle sedi. Nel caso della Lombardia emergono per la varietà delle problematiche analizzate gli articoli sulla morfologia glaciale, sul clima e i temi ambientali. Altre due regioni a cui è dedicato un consistente numero di contributi sono la Sicilia e il Lazio. Per quanto riguarda la Sicilia, oltre ad una monografia emergono contributi di geografia fisica e di geografia delle sedi; mentre nel caso del Lazio prevalgono gli studi condotti in ambito locale riuniti in un numero monografico del "Semestrale di Studi e Ricerche di Geografia".

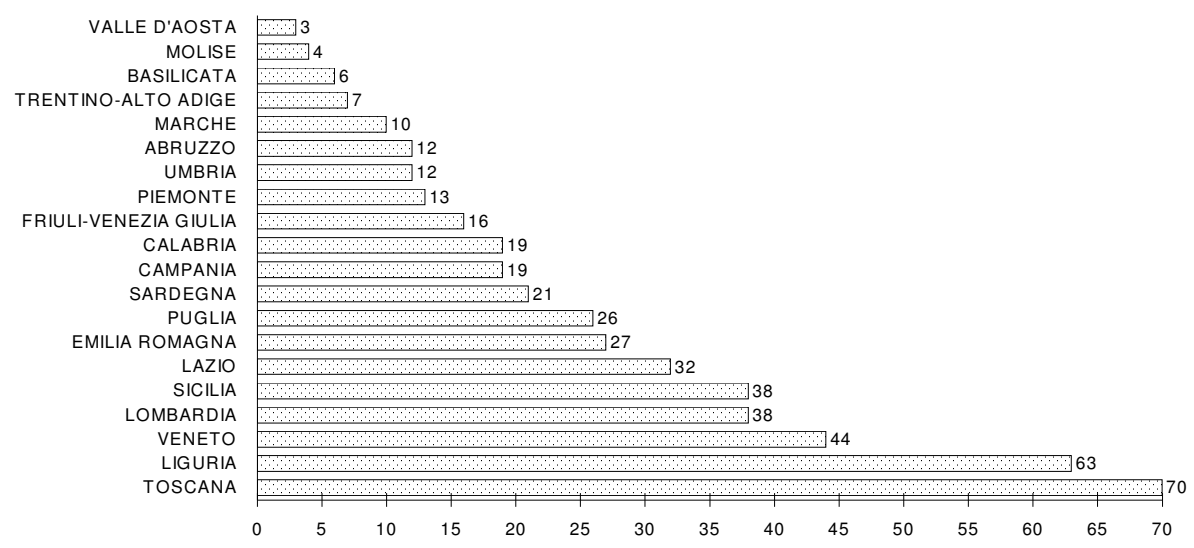

Fig. 4 - Ripartizione degli studi regionali

Figura 4 - Repartição dos estudos regionais

\section{4- CONSIDERAZIONI CONCLUSIVE}

La funzione della geografia è mutata in relazione alle nuove richieste della cultura, dell'economia e della politica, infatti è fortemente aumentata la domanda di analisi del territorio, dei suoi fondamenti fisici, della sua storia, analisi questa necessaria per programmare uno sviluppo razionale, per cercare di organizzare in modo armonico l'uso dell'ambiente senza causare danni ed utilizzare senza spreco le risorse.

Non è un caso che al Congresso Internazionale di Geografia, tenutosi a Washington nel 1992, sia stato dato il titolo "la Geografia è scoprire" poiché la geografia ha oggi il compito di scoprire le ragioni dei fenomeni e delle strutture che determinano le realtà territoriali per cui si può affermare che si è passati da una geografia delle cose ad una geografia dei problemi. 
Vi hanno concorso 3 fattori. Primo, la teoria del sistema generale è molto maturata arricchendosi attraverso riflessioni sull'idea di complessità che ha favorito sempre una più stretta collaborazione tra scienze dalla natura e scienze sociali.

Secondo, la questione ambientale che negli anni ' 80 ha avuto il suo avvio e che ha sensibilizzato la geografia a individuare e studiare insieme ad altre discipline molti dei grandi problemi di una società che ogni giorno produce impatti crescenti sull'ecosistema terra.

Terzo, il concetto di sviluppo sostenibile maturato attraverso il pensiero scientifico dei grandi programmi internazionali sul cambiamento globale, e sviluppatosi in seno alla Conferenza di Rio (1992) su "Ambiente e Sviluppo".

Allo stato attuale del pensiero scientifico va sottolineato come non vi siano concezioni di regione più coerenti con il principio dello sviluppo sostenibile di quanto lo sia la concezione imperniata sul paradigma del sistema generale. Quest'ultimo fattore risulterà determinante nell'avviare un grande cambiamento nella ricerca per cui sarà necessario riflettere sulle recenti tendenze della geografia, sia in rapporto ai progressi del pensiero scientifico, sia per corrispondere a nuovi obiettivi nella gestione dell'ambiente e delle risorse.

In questo contesto sembra inserirsi appieno la più recente produzione geografica italiana, infatti, dalla bibliografia redatta per il primo biennio degli anni '90 emerge una maggiore ampiezza delle tematiche geografiche che interessano i molti problemi dell'organizzazione e dello sviluppo del territorio e dell'ambiente in cui la teoria regionale assumerà una forte legittimazione se sarà sviluppata sulla base di due paradigmi: un paradigma politico (principio dello sviluppo sostenibile) e un paradigma scientifico (sistema generale) (VALlEGA, 1995). Per cui la letteratura geografica appare assai varia ed eterogenea, volta allo studio di problemi diversi, in un orizzonte di interessi molto aperto e a tal proposito risulta sempre attuale il pensiero di Paul ClAVAL: "La geografia non cessa di meravigliare coloro stessi che la praticano" (Claval, 1972).

$* \quad *$

Si segnala, inoltre la compilazione di: Per una bibliografia geografica della Liguria a cura di Fabio BELLATI e Marina CRICENTI, con la collaborazione di Gian Marco UGoLINI, dedicata agli studi pubblicati tra il 1961 e il 1993. Il volume si presenta, da un lato, come un aggiornamento della precedente bibliografia curata nel 1961 da Gaetano FERRo, dall'altro lato riflette gli orientamenti che negli ultimi trent'anni sono emersi nella geografia italiana.

La bibliografia è stata suddivisa, come la precedente, in due parti: la prima riguarda l'intera area regionale, i rapporti tra la Liguria e altre regioni italiane e le interrelazioni che intercorrono a livello provinciale e locale. La seconda parte comprende studi di carattere subregionale e locale, rivolgendo particolare attenzione alla città di Genova.

Tenendo conto dell'evoluzione avvenuta negli studi geografici e delle caratteristiche peculiari della Liguria è stata compiuta una precisa selezione degli scritti specialistici in campo fisico e naturalistico, che hanno ormai assunto una fisionomia autonoma. È stato conferito ampio spazio agli studi di geografia storica e di storia della cartografia. Nell'ambito delle ricerche di demografia è stato posto l'accento sulle correnti migratorie in partenza dalla Liguria e nel settore dedicato alle attività economiche sono stati evidenziati soprattutto gli scritti che mettono in risalto le problematiche e che contengono aspetti comparativi. Inoltre, è stato riservato ampio 
spazio alle più recenti tematiche geografiche relative all'organizzazione territoriale, alle condizioni dell'ambiente e alla sua protezione.

\section{RIFERIMENTI BIBLIOGRAFICI}

A.Ge.I. (1992) - Atti preparatori del XXVI Congresso Geografico Italiano. Tip. Brigati, Genova.

Bellati, F.; M. CRICENTI (a cura di) (1995) - Per una bibliografia geografica della Liguria (1961-1993). Tip. Brigati, Genova.

BellenCin MeNeghel, G. (a cura di) (1991) - Agriturismo in Italia. Patron, Bologna.

BENCARDINO, F. (a cura di) (1991) - La cartografia geografica nel progresso delle conoscenze sull'Oriente nell'Europa dei secoli XV-XIX - Atti del Convegno. Contributi Geografici, Ist. Univ. Orientale, Napoli.

Bertuglia, C.; La Bella A. (e cura di) (1991) - Y sistemi urbani. Vol. I Le teorie. Il sistema e le reti. F. Angeli, Milano.

BuZzetTI, L. (1992) - Paesaggio; Regione. in M. LAENG (a cura di). Enciclopedia pedagogica, La Scuola, Brescia: 8653-8660; 9871-9878.

CIACCIO, C. (1987) - Temi di geografia del turismo e del tempo libero. in G. CORNA PELLEGRINI (a cura di). Aspetti e problemi della geografia, vol. I, Marzorati, Milano: 599-634.

Citarella, F. (a cura di) (1992) - Emigrazione e presenza italiana in Argentina - Atti del Congresso Internazionale. C.N.R., Roma.

Claval, P. (1972) - L'evoluzione storica della Geografia Umana. F. Angeli, Milano.

COPPOLA, P. (1992) - Diversità della geografia, geografia delle diversità. in G. CORNA PELLEGRINI; E. BIANCHI (a cura di). Varietà delle geografie. Limiti e forza della disciplina, Cisalpino, Milano: 93-97.

CoRna PellegrinI, G. (a cura di) (1987/a) - Aspetti e problemi della geografia. Vol. I, Marzorati, Milano.

Corna Pellegrini, G. (1987/b) - Perché la geografia oggi. in G. CoRna Pellegrini (a cura di). Aspetti e problemi della geografia, vol. I, Marzorati, Milano: 1-11.

CORNA PEllegrinI, G.; E. BIANCHI (a cura di) (1992) - Varietà delle geografie. Limiti e forza della disciplina. Cisalpino, Milano.

DA Pozzo, C. (1992) - Bioetica e geografia: un'interazione possibile. Riv. Geogr. Ital., 3: 503-513.

DEMATTEIS, G. (e cura di) (1992) - Il fenomeno urbano in Italia: interpretazioni, prospettive, politiche. F. Angeli, Milano.

Distaso, S. (a cura di) (1992) - La popolazione delle città italiane. Tendenze in atto e prospettive Atti del Convegno. Cacucci Editore, Bari.

MinCA, C. (1991) - Il waterfront: una nuova frontiera urbana. Porto e Mare, 5/6: 49-54.

NICE, B. (1992) - Scritti geografici. Giunti, Firenze.

ORIOLES, V. (a cura di) (1992) - Studi in memoria di Giorgio Valussi. Dell'Orso, Alessandria.

PAlAgiano, C. (1987) - La geografia medica. in G. CORNA Pellegrini (a cura di). Aspetti e problemi della geografia, vol. I, Marzorati, Milano: 465-508.

Palagiano, C.; G. De Santis; M. G. SCIFONi (a cura di) (1992) - La geografia medica e gli ecosistemi - Atti del IV Seminario Internazionale di Geografia Medica. RUX Editrice, Perugia.

PAlAgiano, C.; E. PARATORE (1991) - Studi in onore di Osvaldo Baldacci, $i$ contenuti geografici a servizio dell'uomo. Vol. II, Patron, Bologna.

Palagiano, C.; E. PARATORe (1991) - Studi in onore di Osvaldo Baldacci, la lettura geografica, il linguaggio geografico a servizio dell'uomo. Vol. I, Patron, Bologna.

Sauro U.; A. Bondesan; M. Meneghel (a cura di) (1991) - Proceedings of the International Conference on Environmental Changes in Karst Areas. I.C.E.C.K.A., Q. Dip., 13, Padova.

SodANo, G. (1992) - La tutela dell'ambiente marino. Riv. Mar., 5: 169-178.

SPOTO, M.; C. FRANZOSINI (1991) - The Natural Marine Reserve of Miramare (Trieste, Italy): Tourism and Environmental Education. Ocean \& Coastal Management, 1: 53-60.

T.C.I. (1991) - Guida d'Italia. Natura Ambiente Paesaggio. T.C.I., Milano.

VALLEGA, A. (1986) - Discorso sul metodo. Riv. Geogr. Ital., 93: 253-283.

Vallega, A. (1990) - Esistenza, società, ecosistema. Pensiero geografico e questione ambientale. Mursia, Milano. 
VALLEGA, A. (1992) - La gestione del mare. Il binomio "Sviluppo e protezione ambientale". in La difesa del mare e delle coste. Metodologie obiettivi attività, Consorzio Pelagos e Ministero marina Mercantile, Roma: 127-134.

VALLEGA, A. (1995) - La regione sistema territoriale sostenibile. Compendio di geografia regionale sistematica. Mursia, Milano.

VARANI, N. (1991) - Aspetti geografici delle aree marine protette: il caso del Mar Ligure. in A. Vallega et al. (a cura di). La Liguria e il Mare, Pubbl. Ist. Sc. Geogr. Univ. Genova, XLV, Genova: 63-97.

VARANI, N.; A. PRIMI (1995) - Bibliografia Geografica della Regione Italiana (anni 1991-1992). Società Geografica Italiana, Roma.

ZuniCA, M. (1992) - Ambiente costiero e valutazione d'impatto. Patron, Bologna. 